\title{
Acute interstitial pneumonitis requiring extracorporeal membrane oxygenation and lung transplantation in an adolescent patient
}

\author{
John R. Spratt, MD, MA, ${ }^{a}$ Emilian Racila, MD, ${ }^{b}$ and Sara J. Shumway, MD, ${ }^{\mathrm{c}}$ Minneapolis, Minn
}

\footnotetext{
From the Departments of a Surgery and ${ }^{\mathrm{b}}$ Laboratory Medicine and Pathology, and ${ }^{\mathrm{c}}$ Division of Cardiothoracic Surgery, Department of Surgery, University of Minnesota, Minneapolis, Minn.

Disclosures: Authors have nothing to disclose with regard to commercial support.

Received for publication June 12, 2017; revisions received July 15, 2017; accepted for publication Aug 11, 2017; available ahead of print Sept 28, 2017.

Address for reprints: Sara J. Shumway, MD, Division of Cardiothoracic Surgery, Department of Surgery University of Minnesota Medical School, 420 Delaware St SE, Mayo Mail Code 195, Minneapolis, MN 55455 (E-mail: shumw001@umn.edu).

J Thorac Cardiovasc Surg 2017;154:e125-7

$0022-5223 / \$ 36.00$

Copyright (C) 2017 by The American Association for Thoracic Surgery

http://dx.doi.org/10.1016/j.jtcvs.2017.08.027
}

Acute interstitial pneumonitis (AIP) is a diagnosis of exclusion characterized by dyspnea and cough following an etiologically ambiguous prodome without prior lung disease progressing to fulminant respiratory failure requiring mechanical ventilation. Management is generally supportive. The prognosis for patients with AIP-related respiratory failure is dismal, with an estimated mortality of $50 \%{ }^{1,2}$ We report an adolescent patient who suffered fulminant AIP managed with extracorporeal membrane oxygenation (ECMO) and eventual bilateral lung transplantation.

\section{CLINICAL SUMMARY}

The patient was a previously healthy 19-year-old woman who sought treatment at an outside facility with reports of shortness of breath and cough. She had a left lower lobe pneumonia diagnosed on the basis of a corresponding

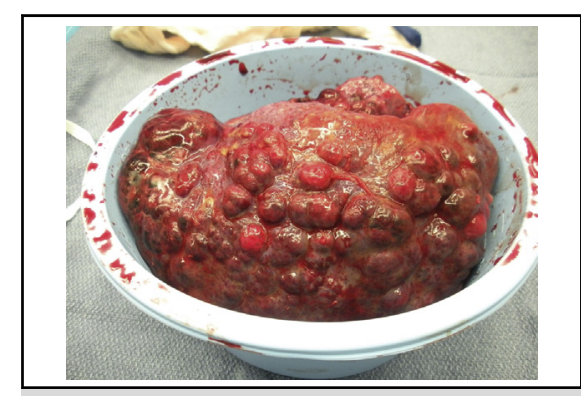

Gross appearance of native lung with rapidly progressive acute interstitial pneumonitis.

\section{Central Message}

Acute interstitial pneumonitis may be rapidly progressive and require advanced therapies, even in young patients. ECMO as a bridge to lung transplantation may be a viable strategy in some cases.

See Editorial Commentary page e129.

radiographic infiltrate (Figure $1, A$ ), and her condition was initially managed with oral antibiotics. Progressive hypoxia developed, necessitating admission 3 days later. At that time, she was found to have worsening infiltrates, which by then were bilateral. The patient required intubation shortly after admission, and severe hypoxia

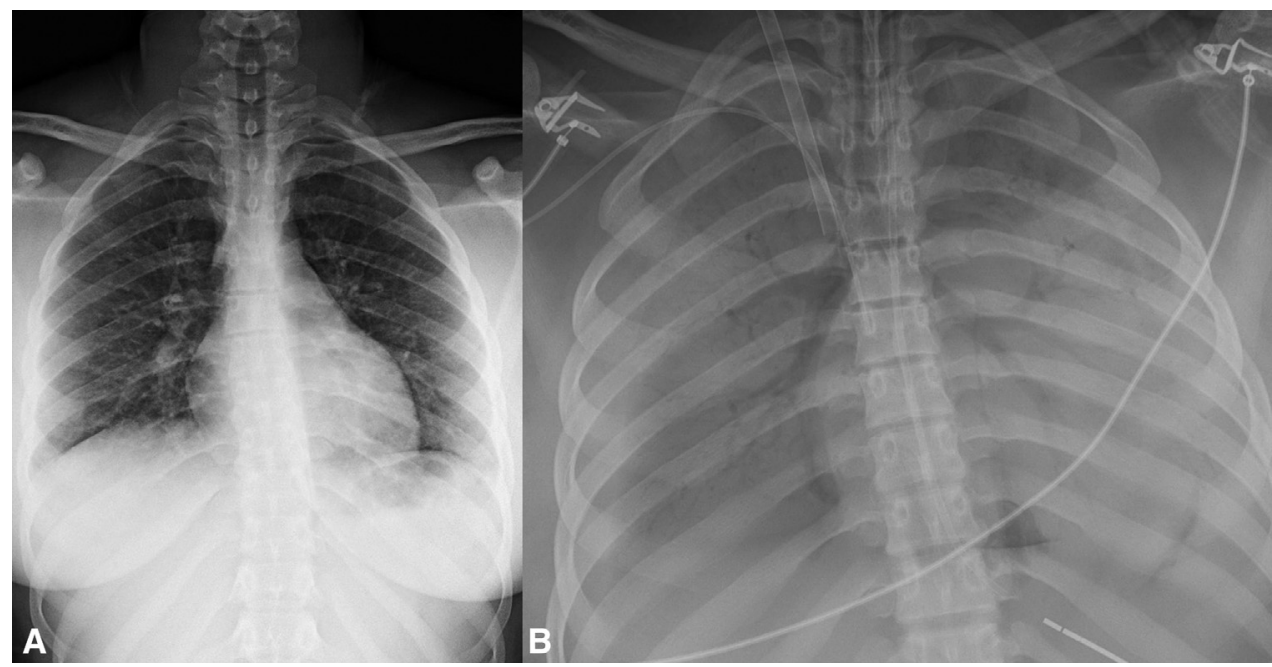

FIGURE 1. A, Chest radiograph at initial presentation. Note mild left lower lobe infiltrate. B, Chest radiograph 10 days later. Note severe bilateral infiltrates with air bronchograms and internal jugular extracorporeal membrane oxygenation cannula. 


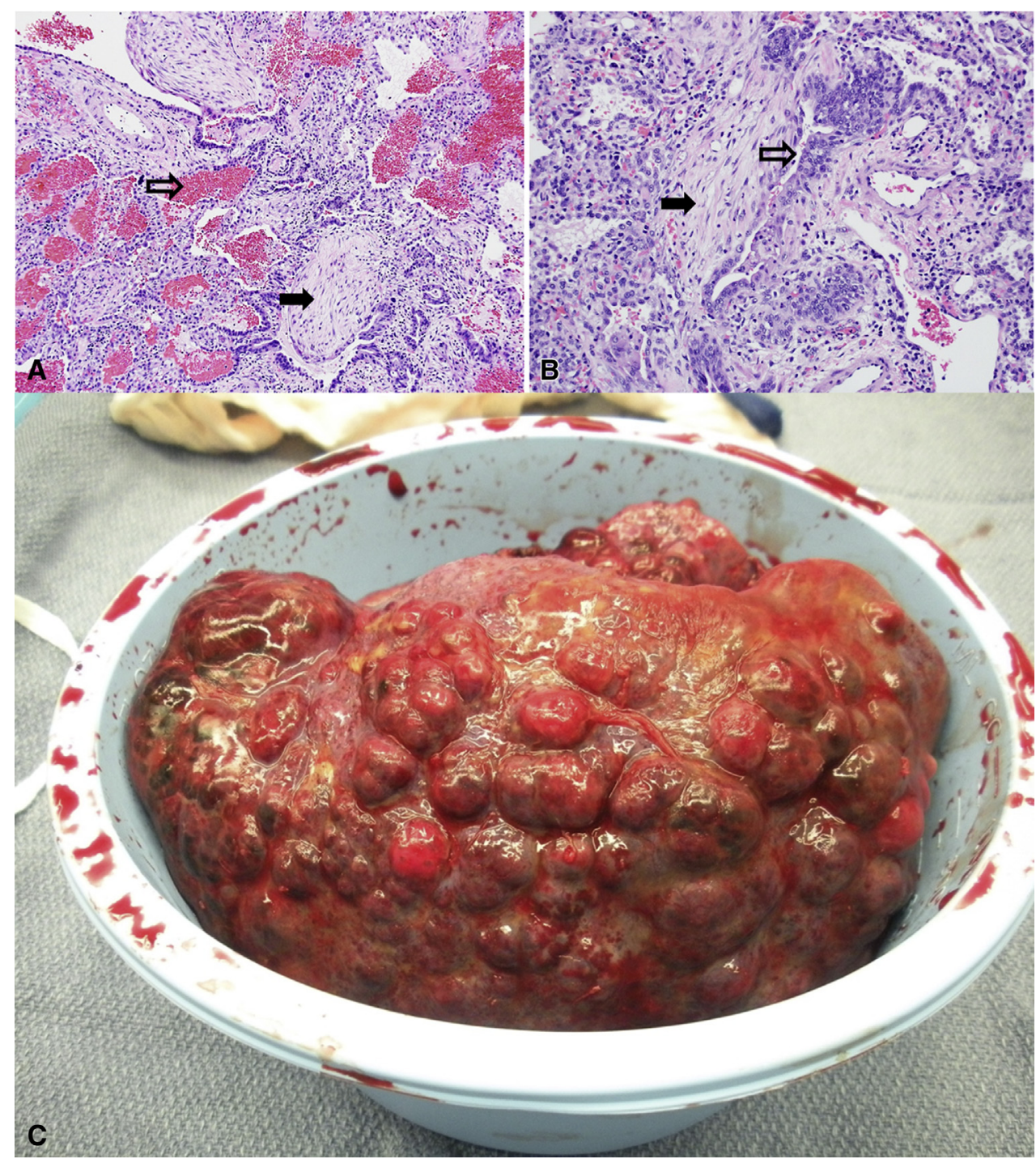

FIGURE 2. A, Microscopic examination of the explanted lung (hematoxylin-eosin stain, 100×) shows features of acute lung injury consisting of Masson bodies of organizing pneumonia (black arrow) and intra-alveolar hemorrhage (open arrow). B, On microscopic examination of the explanted lung (hematoxylin-eosin stain, 200×), bronchiolitis obliterans was frequently observed, consisting of periairway interstitial fibroblast proliferation (black arrow) with markedly narrowed airway lumen (open arrow). Bronchiolitis obliterans and organizing pneumonia together are the components of cryptogenic organizing pneumonia; in this case, however, they are an integrated part of acute lung injury that diffusely involves all lung lobes. C, Gross appearance of explanted lung with obvious alteration of pleural surface by vesicular bullae, pleural adhesions, and fibrinous exudate.

subsequently developed, necessitating paralysis and prone positioning during the next 2 days. This hypoxia was initially thought to be due either to complications of community-acquired pneumonia (on the basis of initial complaints and chest radiography) or an adenoviral infection (on the basis of diarrhea on admission and previous exposure to small children). An extensive workup was performed for bacterial infection (including Legionella, Chlamydia, Mycoplasma, Leptospira, Bartonella henselae, Bordetella pertussis, and acid-fast bacilli), viral infection (including respiratory syncytial virus, human immunodeficiency virus, influenza, hantavirus, and enterovirus), and fungal infection (including Aspergillus,
Pneumocystis, and Histoplasma). Other workup included serum testing for antinuclear, fluorescent antinuclear, and anti-glomerular basement membrane antibodies; testing for C-antineutrophil cytoplasmic antibody and Pantineutrophil cytoplasmic antibody; a hypersensitivity panel; and immune deficiency testing. These tests were unrevealing, prompting a diagnosis of AIP. Anuric renal failure also developed during this time, necessitating renal replacement therapy.

The patient's respiratory status continued to deteriorate and, on ventilator day 6, venovenous ECMO was initiated by our team through the right internal jugular and left femoral veins, after which the patient was transported to 
our facility. Figure 1, $B$, demonstrates her severe bilateral infiltrates at transfer. She continued to demonstrate marginal oxygenation $\left(\mathrm{PaO}_{2} 30-40 \mathrm{~mm} \mathrm{Hg}\right)$ during the ensuing week and had no response to a course of corticosteroids. Bilateral spontaneous pneumothoraxes developed, requiring chest tube placement on ventilator days 11 and 12. Renal replacement therapy was continued. The patient was evaluated by our lung transplant team shortly after admission and was initially thought to be disqualified because of her renal failure and habitus (body mass index, $32.7 \mathrm{~kg} / \mathrm{m}^{2}$ ) at presentation, but this decision was reconsidered because of her young age and lack of respiratory improvement despite ECMO.

The patient was listed for transplant on ventilator day 14 and underwent a bilateral sequential lung transplant with cardiopulmonary bypass and ECMO decannulation on ventilator day 16. ECMO support was weaned at the end of the procedure, but the chest was initially left open because of the potential for cardiac compression by edematous and hyperinflated allografts. The patient required stapled pneumoreduction of the transplanted lingula but otherwise tolerated chest closure on postoperative day 4. After a period of prolonged convalescence and ventilatory weaning, she was discharged to acute rehabilitation approximately 2.5 months after surgery. The patient underwent a living-donor kidney transplant 5 months after discharge and is alive and doing well approximately 2 years after her lung transplant.

\section{DISCUSSION}

\section{Pathology of Explanted Native Lungs}

Microscopic examination revealed extensive acute lung injury, consisting of organizing pneumonia, diffuse alveolar hemorrhage, and patchy proliferative phase of diffuse alveolar damage. Changes of bronchiolitis obliterans were frequently observed, in some areas associated with organizing pneumonia and suggestive of cryptogenic organizing pneumonia (formerly known as bronchiolitis obliterans/organizing pneumonia; Figure 2, $A$ and $B$ ). No signs of acute or chronic infection were identified. Gross examination of the native lungs showed severe bullous and fibrotic changes (Figure 2, C).

\section{ECMO and Lung Transplantation for AIP}

AIP is characterized by dyspnea and cough after a brief, flulike prodrome with no apparent cause in a patient without previous lung disease, which progresses rapidly to fulminant respiratory failure. It is radiographically similar to adult respiratory distress syndrome. Treatment is generally supportive, but the overall prognosis is dismal. ${ }^{1}$ Although AIP is most commonly seen in adults, there are reports of AIP in older children and young adults. ${ }^{1-3}$ Other descriptions of advanced therapy for AIP are limited.

AIP must be considered when evaluating severe respiratory failure without apparent cause in patients of all ages. The overall prognosis is poor. There is growing experience with ECMO, however, even when initiated distant from the transplant center, as a bridge to lung transplantation for fulminant end-stage pulmonary disease. ${ }^{4,5}$ The either or both these therapies may benefit such patients. Early referral to transplant centers is warranted for patients with rapidly progressive disease.

\section{References}

1. Bouros D, Nicholson AC, Polychronopoulos V, du Bois RM. Acute interstitial pneumonia. Eur Respir J. 2000;15:412-8.

2. Turillazzi E, Di Donato S, Neri M, Riezzo I, Fineschi V. An immunohistochemical study in a fatal case of acute interstitial pneumonitis (Hamman-Rich syndrome) in a 15-year-old boy presenting as sudden death. Forensic Sci Int. 2007; $173: 73-7$

3. Turner DA, Rehder KJ, Bonadonna D, Gray A, Lin S, Zaas D, et al. Ambulatory ECMO as a bridge to lung transplant in a previously well pediatric patient with ARDS. Pediatrics. 2014;134:e583-5.

4. Javidfar J, Brodie D, Iribarne A, Jurado J, Lavelle M, Brenner K, et al. Extracorporeal membrane oxygenation as a bridge to lung transplantation and recovery. $J$ Thorac Cardiovasc Surg. 2012;144:716-21.

5. Hoopes CW, Kukreja J, Golden J, Davenport DL, Diaz-Guzman E, Zwischenberger JB. Extracorporeal membrane oxygenation as a bridge to pulmonary transplantation. J Thorac Cardiovasc Surg. 2013;145:862-7; discussion $867-8$. 